\title{
Structural evolution and skyrmionic phase diagram of the lacunar spinel $\mathrm{GaMo}_{4} \mathrm{Se}_{8}$
}

\author{
Emily C. Schueller, ${ }^{1}$ Daniil A. Kitchaev, ${ }^{1}$ Julia L. Zuo, ${ }^{1}$ Joshua D. Bocarsly, ${ }^{1}$ \\ Joya A. Cooley, ${ }^{1}$ Anton Van der Ven, ${ }^{1}$ Stephen D. Wilson, ${ }^{1}$ and Ram Seshadri ${ }^{1}$ \\ ${ }^{1}$ Materials Department and Materials Research Laboratory \\ University of California, Santa Barbara, Santa Barbara, CA, 93106
}

(Dated: May 15, 2020)

\begin{abstract}
In the $A B_{4} Q_{8}$ lacunar spinels, the electronic structure is described on the basis of inter- and intracluster interactions of tetrahedral $B_{4}$ clusters, and tuning these can lead to myriad fascinating electronic and magnetic ground states. In this work, we employ magnetic measurements, synchrotron $\mathrm{X}$-ray and neutron scattering, and first-principles electronic structure calculations to examine the coupling between structural and magnetic phase evolution in $\mathrm{GaMo}_{4} \mathrm{Se}_{8}$, including the emergence of a skyrmionic regime in the magnetic phase diagram. We show that the competition between two distinct Jahn-Teller distortions of the room temperature cubic $F \overline{4} 3 \mathrm{~m}$ structure leads to the coexistence of the ground state $R 3 \mathrm{~m}$ phase and a metastable $I m m 2$ phase. The magnetic properties of these two phases are computationally shown to be very different, with the $I m m 2$ phase exhibiting uniaxial ferromagnetism and the $R 3 \mathrm{~m}$ phase hosting a complex magnetic phase diagram including equilibrium Néel-type skyrmions stable from nearly $T=28 \mathrm{~K}$ down to $T=2 \mathrm{~K}$, the lowest measured temperature. The large change in magnetic behavior induced by a small structural distortion reveals that $\mathrm{GaMo}_{4} \mathrm{Se}_{8}$ is an exciting candidate material for tuning unconventional magnetic properties via mechanical means.
\end{abstract}

\section{INTRODUCTION}

In recent years, there has been an increasing recognition that in some transition metal compounds, metalmetal bonding can often interact with electron correlation resulting in unconventional magnetic and electronic behavior, as well as unusually strong coupling between magnetic, electronic, and structural degrees of freedom [1-4]. An important class of materials where this becomes evident are the lacunar spinels, which are characterized by tetrahedral clusters of transition metal atoms [5]. Two members of the lacunar spinel family, $\mathrm{GaV}_{4} \mathrm{~S}_{8}$ and $\mathrm{GaV}_{4} \mathrm{Se}_{8}$, have garnered significant attention in recent years as multiferroic materials as well as Néeltype skyrmion hosts [6-8]. At low temperatures, these materials crystallize in the polar $R 3 m$ space group [9] and support complex magnetic phase diagrams with an ordered moment of $1 \mu_{B} / \mathrm{V}_{4}$ cluster. The related Mocontaining compounds, $\mathrm{GaMo}_{4} \mathrm{~S}_{8}$ and $\mathrm{GaMo}_{4} \mathrm{Se}_{8}$, have been less studied, although they are reported to have a similar low temperature crystal structure, but with a compressive rather than elongative distortion along the cubic [111] direction [9-11].

Bulk magnetic measurements have revealed similar metamagnetic behavior between the Mo and the Vcontaining compounds [12, 13], while computational analysis of lacunar spinel ferromagnets suggests that all materials with this structure type are likely to host skyrmions across wide temperature ranges [14]. However, because Mo is a heavier element than V, the Mobased compounds exhibit increased spin-orbit coupling, leading to more pronounced magnetic anomalies over greater regions of magnetic field and temperature. Additionally, spin-orbit coupling can affect the electronic properties through splitting of degenerate molecular orbitals, as seen in $\mathrm{GaTa}_{4} \mathrm{Se}_{8}$ [15]. A recent report on $\mathrm{GaMo}_{4} \mathrm{~S}_{8}$ indicates that this material harbors complex modulated magnetic phases and that the increased spinorbit coupling in the material leads to unusual "waving" effects on the magnetic ordering vectors [16].

In this work, we perform a detailed theoretical and experimental study of $\mathrm{GaMo}_{4} \mathrm{Se}_{8}$ in order to characterize the coupling between the crystal structure evolution and magnetic properties. We discover that at the structural phase transition, $\mathrm{GaMo}_{4} \mathrm{Se}_{8}$ can transform into two polar space groups, the reported rhombohedral $R 3 \mathrm{~m}$ phase, which is the ground state, and an orthorhombic Imm2 phase, which is metastable. On the basis of computational and experimental data, we show that the $R 3 \mathrm{~m}$ phase of $\mathrm{GaMo}_{4} \mathrm{Se}_{8}$ hosts a rich magnetic phase diagram with long wavelength magnetic order, including cycloids and Néel-type skyrmions, with periodicities on the order of $16 \mathrm{~nm}$. In contrast, the competing $\operatorname{Imm} 2$ phase is suggested from first-principles calculations to be a strongly uniaxial ferromagnet. The dramatic change in magnetic behavior associated with a subtle change in crystal structure highlights the strong coupling between crystal structure and magnetism in $\mathrm{GaMo}_{4} \mathrm{Se}_{8}$, and suggests an avenue for tuning skyrmion and other exotic magnetic phases via strain.

\section{METHODS}

\section{A. Computational Methods}

All electronic structure calculations were performed using the Vienna Ab-Initio Simulation Package (VASP) [17] with projector-augmented-wave pseudopotentials $[18,19]$ within the Perdew-Burke-Ernzerhof generalized gradient approximation [20]. We do not employ an atom-centered Hubbard- $U$ correction as our previous analysis of the related $\mathrm{GaV}_{4} \mathrm{Se}_{8}$ system showed that this leads to an incorrect high-moment magnetic configura- 
tion by penalizing metal-metal bonding [4]. Structural distortions from the high temperature cubic structure to the low temperature orthorhombic and rhombohedral structures were generated using ISODISTORT [21]. Structural stability calculations used a Gamma-centered $k$-point grid of $8 \times 8 \times 8$ and an energy cutoff of $500 \mathrm{eV}$ with a collinear magnetic configuration of $1 \mu_{B} / \mathrm{Mo}_{4}$. Computational results were parsed and visualized with the python package pymatgen [22]. Noncollinear magnetic calculations accounting for spin-orbit coupling followed the methodology described by Kitchaev et al. [14]. All calculations were performed statically, on the basis of the experimentally determined crystal structures, and converged to $10^{-6} \mathrm{eV}$ in total energy. To reduce noise associated with $k$-point discretization error, all spinconfiguration energies were referenced to the energy of a $c$-axis ferromagnet computed within the same supercell. Noncollinear magnetic configurations were initialized by evenly distributing the magnetic moment of each $\mathrm{Mo}_{4}$ tetrahedron on the four Mo atoms, i.e. as a locally ferromagnetic configuration of $0.25 \mu_{B}$ per Mo. The magnetic moment of each tetrahedron was then obtained from the DFT data by summing the individual magnetic moments projected onto the four Mo atoms.

The magnetic cluster expansion Hamiltonian [14, 2325] used to construct the magnetic phase diagram was derived by considering single-spin and nearest-neighbor pair interactions, as shown in Supplementary Figure S4 [26]. The interaction strengths were parametrized on the basis of collinear and spin-wave enumerations up to a supercell size of 4, as well as several sets of spin configurations iteratively generated to independently constrain the terms in the Hamiltonian.[27] Note that due to the very small energy scale of magnetocrystalline anisotropy, we fit the single-site anisotropy coefficients independently of the pair interactions, by fitting to the energy of rotating the ground-state collinear spin configurations within the primitive cell of the structure, so as to eliminate all noise arising from changes in the $k$-point grid [14]. The resulting parametrization leads to an error below $5 \mu \mathrm{eV} / \mathrm{Mo}_{4}$ for the magnetocrystalline anisotropy, and below $1 \mathrm{meV} / \mathrm{Mo}_{4}$ across all non-collinear spin configurations, as shown in Supplementary Figure S5 [26]. The full Hamiltonians and fitted interaction parameters for the $R 3 \mathrm{~m}$ and $I m m 2$ phases are given in Supplementary Tables 1 and 2 [26].

\section{B. Experimental Methods}

$\mathrm{GaMo}_{4} \mathrm{Se}_{8}$ powder was obtained by reaction of $\mathrm{Ga}$ pieces and ground Mo and Se powders with an approximately $50 \%$ excess of elemental $\mathrm{Ga}$. The elements were reacted in an evacuated fused silica tube in $1 \mathrm{~g}$ batches with a heating ramp rate of $8^{\circ} \mathrm{C} / \mathrm{min}$ to $1010^{\circ} \mathrm{C}$, held for 20 hours, and quenched [28].

High resolution synchrotron powder X-ray diffraction was performed at the Advanced Photon Source at Ar- gonne National Laboratory at the 11-BM beamline. Approximately $30 \mathrm{mg}$ of powder was loaded into a kapton capillary and placed into a cryostat. Temperaturedependent scans with a 10 minute exposure time were taken from $T=10 \mathrm{~K}$ to $T=250 \mathrm{~K}$. Rietveld refinements were performed using the Topas analysis package [29]. Low-temperature structure solution was performed by first fitting the reported rhombohedral structure and then indexing the remaining structural peaks using the EXPO2014 software. The symmetry of the unit cell generated by EXPO was compared to various subgroups of the high temperature $F \overline{4} 3 m$ structure using the ISODISTORT software package [21]. A structure for the corresponding orthorhombic $I m m 2$ space group was generated using ISODISTORT and Rietveld refinements of both the rhombohedral and orthorhombic phases were performed using TOPAS Academic [29]. Due to $h k l$ dependent strain, the Stephens peak shape was used in conjunction with Gaussian size broadening to fit the highly anisotropic peak shapes. Crystal structures were visualized using the VESTA software suite [30].

Small angle neutron scattering measurements were performed at the National Institute of Standards and Technology (NIST) NG-7 neutron beamline. Approximately $100 \mathrm{mg}$ of powder was cold-pressed into a $5 \mathrm{~mm}$ pellet and placed into a cryostat. Exposures were taken over $8 \mathrm{~h}$ durations at various applied magnetic fields with the field applied parallel to the neutron beam. Three scans were taken below the magnetic ordering temperature and a $3 \mathrm{~h}$ background scan was performed with no applied field above the magnetic ordering temperature. The data integration and background subtraction was performed in the IGOR Pro software [31].

Bulk magnetic measurements were performed on a Quantum Design MPMS 3 SQUID Vibrating Sample Magnetometer, with approximately $30 \mathrm{mg}$ of powder loaded into a plastic container and mounted in a brass holder. DC magnetization $v s$. field scans were performed on increasing field and AC susceptibility vs. field measurements were performed on decreasing field. For magnetoentropic mapping measurements, temperaturedependent magnetization measurements from $2 \mathrm{~K}$ to $40 \mathrm{~K}$ were performed with applied magnetic fields varying from 0 Oe to 4000 Oe in increments of 75 Oe. Derivatives and $\Delta S_{m}$ calculations were performed using python code available from Bocarsly et al. [32].

\section{RESULTS}

The room temperature crystal structure of $\mathrm{GaMo}_{4} \mathrm{Se}_{8}$ is highlighted in Figure 1(a), characterized by tetrahedral clusters of Mo atoms which behave like molecular units with a spin of $1 \mu_{B}$ per $\mathrm{Mo}_{4}$ cluster. Through experimental and computational techniques, we find that $\mathrm{GaMo}_{4} \mathrm{Se}_{8}$ follows qualitatively similar behavior to $\mathrm{GaV}_{4} \mathrm{Se}_{8}$, with complex magnetic order arising from a Jahn-Teller distortion of the high-temperature $F \overline{4} 3 \mathrm{~m}$ 


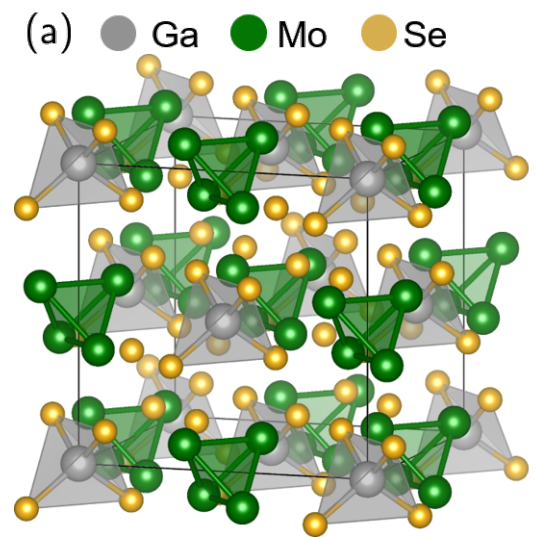

(b)

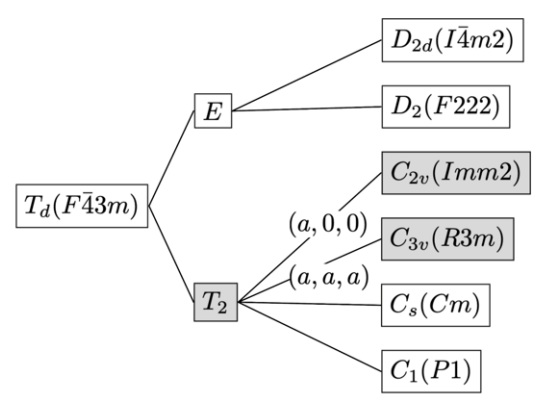

(c)

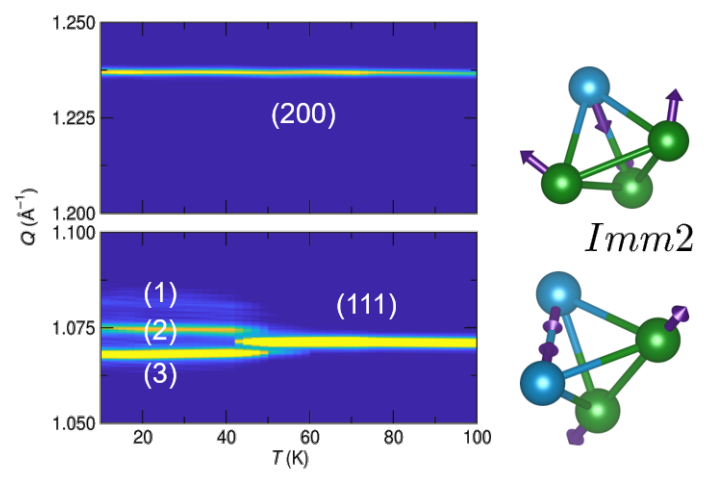

FIG. 1. (a) The cubic $F \overline{4} 3 \mathrm{~m}$ crystal structure of $\mathrm{GaMo}_{4} \mathrm{Se}_{8}$, characterized by tetrahedral clusters of Mo atoms. (b) All subgroups of $F \overline{4} 3 m$ which keep the primitive unit cell volume the same. $R 3 m$ and $I m m 2$ both follow the $T_{2}$ irrep but through different order parameter directions. (c) The evolution of peaks upon cooling in synchrotron XRD data. At $50 \mathrm{~K}$ the (111) cubic peak splits into 3 while the (200) cubic peak stays undistorted. Because the middle peak of the (111) split shifts relative to the peak in the cubic phase while the (200) peak does not shift, it is not possible to explain the low temperature structure by a combination of the cubic phase and a single low temperature phase. (d) The distortion of the $\mathrm{Mo}_{4}$ tetrahedron as it goes into the $R 3 \mathrm{~m}$ and $I m m 2$ space groups. Both are polar, but the $\operatorname{Im} m 2$ cluster has lower symmetry, with three unique bond lengths, compared to two in the $R 3 m$ cluster.

structure. However, in addition to the $R 3 \mathrm{~m}$ ground state, $\mathrm{GaMo}_{4} \mathrm{Se}_{8}$ forms a competing metastable $\operatorname{Imm} 2$ phase, defined by an orthorhombic Jahn-Teller distortion and distinctly different magnetic properties. We first characterize the formation and coexistence of these two structures, and then establish how the character of the lowtemperature distortion controls the magnetic phase diagram of each phase.

Figure 1(c) shows high-resolution synchrotron powder diffraction data as a function of temperature. At $T=50 \mathrm{~K}$, the (111) cubic peak splits into 3 peaks, with the two outer peaks corresponding to an $R 3 \mathrm{~m}$ distortion. Previous X-ray diffraction studies of the structural phase transition of $\mathrm{GaMo}_{4} \mathrm{Se}_{8}$ have rationalized the presence of extra peaks below the transition temperature $(T=51 \mathrm{~K})$ as the coexistence of the high temperature cubic $F \overline{4} 3 \mathrm{~m}$ phase and the rhombohedral $R 3 \mathrm{~m}$ phase [10]. However, with high-resolution measurements employed here, we find that the low-temperature data cannot be fit by a combination of the $R 3 \mathrm{~m}$ phase and the $F \overline{4} 3 \mathrm{~m}$ phase. The middle peak shown in the lower panel of Figure 1(c), which was previously assumed to be a continuation of the cubic $(F \overline{4} 3 m)$ phase, shifts significantly at the phase transition, while the higher $Q$ peak [cubic (200)] shown in the top panel does not split or shift at the phase transition. Because there is only one lattice parameter in the cubic phase, it cannot support a shift of one peak but not another, meaning that there must be another explanation for the additional peaks.

We performed a search for a lower symmetry subgroup of $R 3 m$ (such as a monoclinic $C 2 / m$ phase) that could explain the additional peaks, but found none that did not add add extraneous peaks, not present in the data. We indexed the peaks that could not be explained by the $R 3 m$ unit cell and found they corresponded to an orthorhombic, polar $\operatorname{Imm} 2$ phase. $\operatorname{Imm} 2$, while a subgroup of the parent $F \overline{4} 3 \mathrm{~m}$ structure, is not a subgroup of the $R 3 \mathrm{~m}$ space group. The transition pathways from the $F \overline{4} 3 \mathrm{~m}$ phase to the $R 3 \mathrm{~m}$ and $I m m 2$ phases are associated with two different order parameter directions, but with the same irreducible representation $\left(T_{2}\right)$ as shown in Figure 1(c), indicating the two subgroups are symmetrically similar. This similarity can be seen in the diffraction data; both low temperature space groups have diffraction patterns characterized by splitting of the same cubic peaks, but the new peaks are distinct in number and position between the two phases. The difference between the two structures is most obvious in the distortion of the $\mathrm{Mo}_{4}$ tetrahedron through the phase transition, indicated by the arrows in Figure 1(d), which leads to 2 sets of Mo-Mo bond lengths in the $R 3 \mathrm{~m}$ phase and 3 sets in the $I m m 2$ phase.

It is clear from the diffraction data that the $R 3 \mathrm{~m}$ and Imm 2 phases form simultaneously and coexist in the powder. However, the structures are noticeably strained, as evidenced by the broad line shapes, and have a large amount of peak overlap due to their similarity, which introduces uncertainty in the determination of relative weight percentages of the two phases. As seen in Figure 2(a), our best fit to the data at $T=10 \mathrm{~K}$ yields about $30 \%$ of the orthorhombic phase and $70 \%$ of the rhombohedral phase. In order to fit the unusual peak shapes, we use two phases for the $R 3 \mathrm{~m}$ structure, one with small particles (strong effects of size broadening) and one with large particles, both with strain effects. The $I m m 2$ phase is fit with $h k l$-dependent strain broadening using the Stephens peak-shape. This combination of phases to describe a single peak is shown in Figure 2(b). At $T=50 \mathrm{~K}$, the diffraction data is best fit by a combination of the cubic phase and both the $R 3 \mathrm{~m}$ and $I m m 2$ phases, in- 


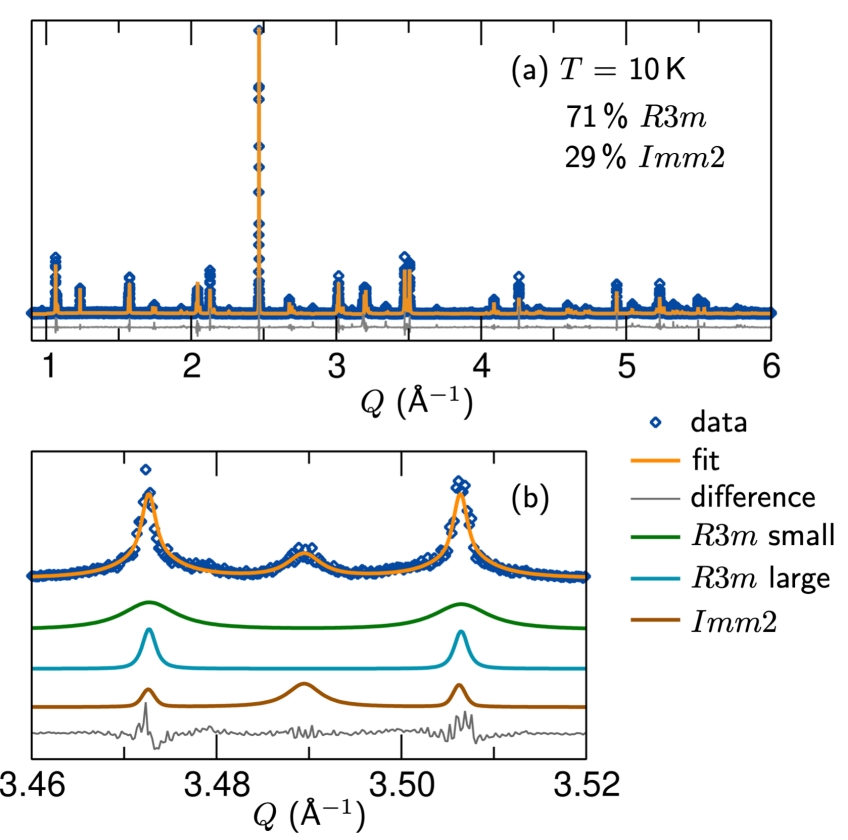

FIG. 2. (a) At $10 \mathrm{~K}$, there is about $70 \%$ of the rhombohedral phase and $30 \%$ of the orthorhombic phase. (b) The complex peak shapes can be fit with two $R 3 m$ phases with different particle sizes, and an $I m m 2$ phase with $h k l$-dependent strain broadening.

dicating both phases form simultaneously at the phase transition temperature and there is no phase region with only one of the two low temperature structures. A similar phase transition from a high symmetry structure into two subgroups has been reported in the oxide spinels $\mathrm{MgCr}_{2} \mathrm{O}_{4}$ and $\mathrm{ZnCr}_{2} \mathrm{O}_{4}$ and was attributed to internal strain creating a metastable and stable phase [33].

To probe the energy landscape for these two phases, we turn to density functional theory (DFT) calculations. We find that while the $R 3 \mathrm{~m}$ phase is the ground state structure, its formation may be kinetically suppressed relative to the $I m m 2$ phase. Using ISODISTORT, we generated distorted structures along the path from the $F \overline{4} 3 \mathrm{~m}$ phase to the $R 3 \mathrm{~m}$ phase and the $I m m 2$ phase. These calculations show that the orthorhombic structure is less stable than the rhombohedral structure by about $20 \mathrm{meV} / \mathrm{Mo}_{4}$ cluster, as shown in Figure 3(a). However, the transition pathway leading to the $R 3 \mathrm{~m}$ phase proceeds over an energy barrier, while the formation of the Imm 2 phase has a smaller barrier. Furthermore, we observe experimentally that the percentage of the $R 3 \mathrm{~m}$ phase increases with decreasing temperature, as can be seen in Figure 3(b). Thus, both computation and experiments indicate that the $R 3 \mathrm{~m}$ arrangement is the likely ground state. Nonetheless, it is possible that due to the low temperature of the phase transition $(T=51 \mathrm{~K})$, the material could get trapped in the orthorhombic structure and lack the energy to convert to the rhombohedral structure. It is also likely that strain effects impact the energy landscape of the two phases and could stabilize
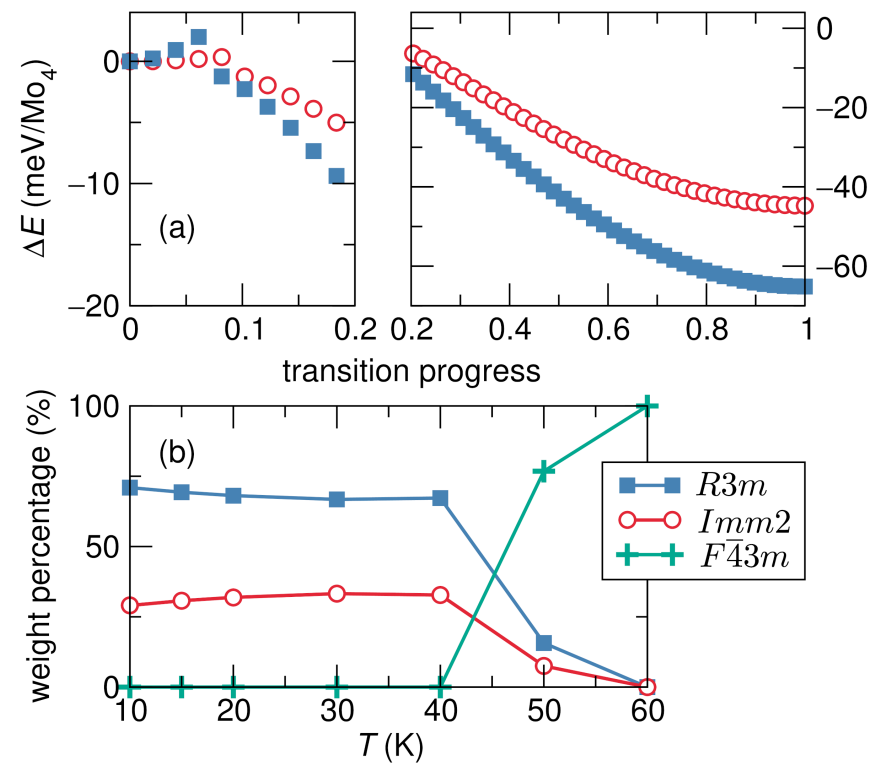

FIG. 3. (a) DFT energies along the transition pathways from the cubic phase to the rhombohedral and orthorhombic phases. The $R 3 \mathrm{~m}$ structure has lower energy, making it the ground state structure, but it has a higher formation energy barrier, highlighted in the left panel. The energy difference is $20 \mathrm{meV} / \mathrm{Mo}_{4}$ cluster. (b) The phase evolution of $\mathrm{GaMo}_{4} \mathrm{Se}_{8}$. Upon cooling, the percentage of the $R 3 \mathrm{~m}$ phase slightly increases, indicating it is likely the ground state crystal structure.

the $\operatorname{Imm} 2$ phase.

Analogous to other lacunar spinels, the magnetic behavior of $\mathrm{GaMo}_{4} \mathrm{Se}_{8}$ is closely tied to its crystal structure. Temperature-dependent magnetization data reveals largely ferromagnetic ordering with an ordering temperature of approximately $T=27.5 \mathrm{~K}$, in agreement with previous reports [13]. $\mathrm{GaMo}_{4} \mathrm{Se}_{8}$ has a saturation magnetization of close to $1 \mu_{B} / \mathrm{Mo}_{4}$ cluster, as shown in Figure 4(a), in agreement with literature [13] and molecular orbital theory. However, field-dependent magnetization measurements show the presence of multiple magnetic phase transitions at all temperatures below the ordering temperature down to $T=2 \mathrm{~K}$, as shown in Figure 4(b). Resolving the nature of these phase transitions is complicated by the fact that by analogy to Vbased lacunar spinels, it is likely that the magnetic phase diagram of $\mathrm{GaMo}_{4} \mathrm{Se}_{8}$ depends strongly on the direction at which the magnetic field is oriented relative to the polar axis of the crystal. As all of our data is collected on a powder sample under uniaxial applied magnetic field, different grains can have different magnetic phases, complicating the interpretation of bulk magnetization data. Nonetheless, by combining a variety of experimental and computational signatures of magnetic phase stability, we are able to understand the observed magnetic phase transitions and confirm the existence of a skyrmion phase.

A recently presented experimental technique for resolving magnetic phase diagrams is magnetoentropic 


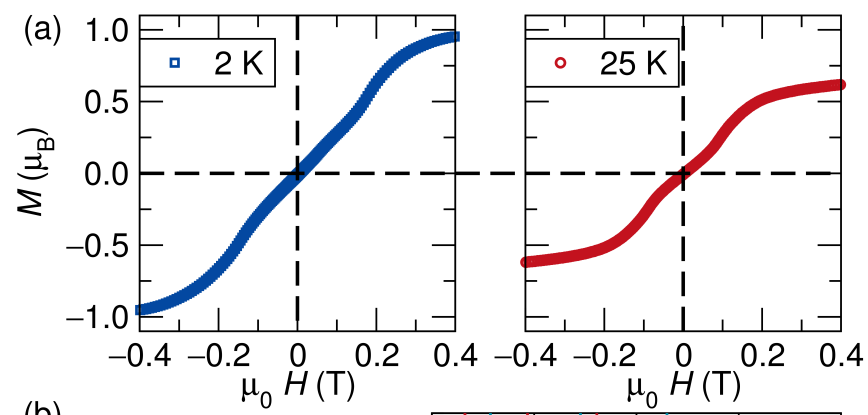

(b)

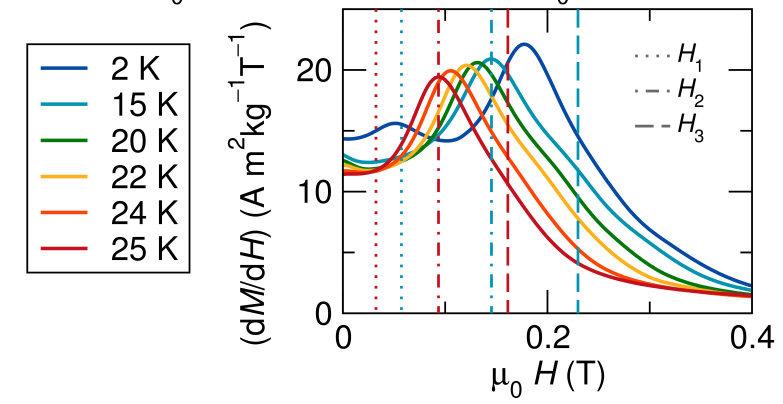

FIG. 4. (a) At $T=2 \mathrm{~K}$, the saturation magnetization is $1 \mu_{B} / \mathrm{Mo}_{4}$ cluster, in agreement with DFT and molecular orbital theory. Multiple magnetic phase transitions are evident in fielddependent magnetization measurements. (b) Phase transitions are shown more clearly in the derivative of magnetization with respect to field. These transitions persist down to $T=2 \mathrm{~K}$, the lowest temperature measured.

mapping. This technique [32], relies on the Maxwell relation that $(\partial M / \partial T)_{H}=(\partial S / \partial H)_{T}$ to identify field driven magnetic phase transitions that are associated with a change in magnetic entropy. In particular, skyrmions form as a high entropy phase, so that the formation of skyrmions is typically associated with a positive entropy anomaly $[32,34,35]$. On a map of $(\partial S / \partial H)_{T}$, shown in Figure 5(b), generated from temperature-dependent-magnetization measurements at various applied fields, shown in Supplementary Figure S3 [26], we can see a red positive entropy anomaly that starts near the Curie temperature and broadens upon cooling. Overlaying this data with peaks in DC susceptibilities $(\partial M / \partial T)$, shown in Supplementary Figure S1 [26], and $(\partial M / \partial H)$ vs. $H$ curves, shown in Figure 4(b), as well as AC susceptibility measurements, shown in Supplementary Figure S2 [26], we observe these signatures are in agreement with the transitions from the entropy map. From these measurements we can generate the phase diagram shown in Figure 5(a) which suggests that the high-entropy region corresponds to a skyrmion phase with behavior similar to that seen in $\mathrm{GaV}_{4} \mathrm{Se}_{8}$. The vertical portion of the entropy anomaly near the Curie temperature has been observed in other skyrmion host materials [32] and has been posited to be a Brazovskii transition into a short-range-ordered or fluctuation-disordered (fd) magnetic phase.

Susceptibility data reveals two additional transitions not visible in the magnetoentropic map, indicating the
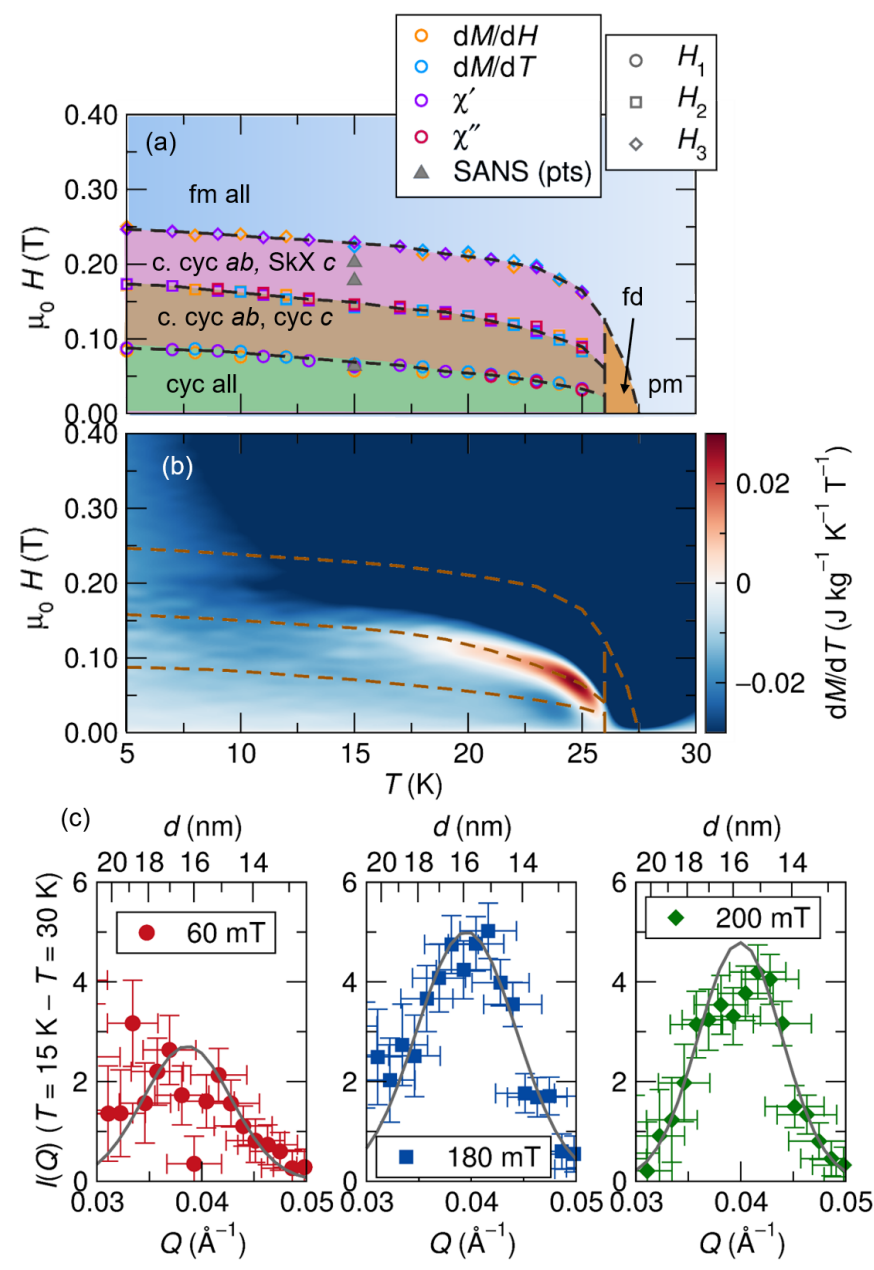

FIG. 5. (a) Using multiple bulk magnetization measurement techniques, we suggest a field $v s$. temperature phase diagram for $\mathrm{GaMo}_{4} \mathrm{Se}_{8}$. Based on theoretical calculations and the phase diagrams for the related V-compounds, we posit that the low field transition is a transition from cycloidal into canted cycloid or ferromagnetic ordering in grains (denoted $a b$ ) where the field is mostly perpendicular to the polar axis. Upon increasing field, grains (denoted $c$ ) where the field is mostly aligned with the polar axis transition into a skyrmion phase and then become field polarized. Near the Curie temperature there is a fluctuation-disordered (fd) phase. (b) The transition into the proposed skyrmion phase in $c$-oriented grains is associated with an increase in magnetic entropy, shown as a red feature near the Curie temperature that broadens upon cooling. (c) SANS data shows the presence of approximately $16 \mathrm{~nm}$ magnetic periodicity at multiple applied fields at $T=15 \mathrm{~K}$. The red points are in the cycloidal phase and the blue and green are in the skyrmion phase. The gray lines are Gaussian fits to guide the eye.

presence of phase transitions not associated with a large change in magnetic entropy. To unambiguously resolve these phase transitions, as well as to disentangle the contributions of the $R 3 \mathrm{~m}$ and $I m m 2$ phases in the powder, we turn to computational modeling. Our computational analysis relies on a magnetic cluster expansion 


\begin{tabular}{lcccc}
\hline \hline & $\begin{array}{c}A \\
\left(10^{-13} \mathrm{~J} / \mathrm{m}\right)\end{array}$ & $\begin{array}{c}D_{x y} \\
\left(10^{-4} \mathrm{~J} / \mathrm{m}^{2}\right)\end{array}$ & $\begin{array}{c}D_{u} \\
\left(10^{4} \mathrm{~J} / \mathrm{m}^{3}\right)\end{array}$ \\
\hline$R 3 m$ & 3.48 & -2.35 & 2.35 & 7.34 \\
Imm2 & 4.15 & -5.00 & -0.682 & -182 \\
\hline \hline
\end{tabular}

TABLE I. Effective micromagnetic interaction parameters in the low-temperature limit for the $R 3 \mathrm{~m}$ and $I m m 2$ phases of $\mathrm{GaMo}_{4} \mathrm{Se}_{8}$. See main text and Supplementary Figure S4 [26] for a definition of variables and coordinate systems.

Hamiltonian parameterized using density functional theory (DFT), which has been recently demonstrated to yield reliable magnetic phase diagrams for the lacunar spinels [14]. We are thus able to independently resolve the anisotropic magnetic phase diagrams expected in the $R 3 m$ and $I m m 2$ phases and rationalize the observed magnetic behavior of the biphasic powder.

First, we demonstrate that the zero-field, lowtemperature ground state magnetic configuration of the $R 3 \mathrm{~m}$ phase is a cycloid with a predicted wavelength of $18 \mathrm{~nm}$, while the $\operatorname{Imm} 2$ phase is a uniaxial ferromagnet. Figure 6(a) maps out the energy of the ferromagnetic state in both phases relative to the energy of the ground state magnetic phase. The $I m m 2$ phase strongly favors magnetization along the $b$-axis, parallel to the shortest Mo-Mo bond. The $R 3 \mathrm{~m}$ phase exhibits a predominantly easy-plane anisotropy, normal to the compressive distortion of the $\mathrm{Mo}_{4}$ tetrahedron, with a three-fold modulation favoring spin orientation towards the corners of the tetrahedron. While the Dzyaloshinskii-Moriya interaction (DMI) in the two phases is comparable in magnitude and favors cycloidal spin textures in the $(a b)-$ planes [36], the large magnetocrystalline anisotropy of the $I m m 2$ phase suppresses any spin configuration deviating from the $b$-axis, forcing a ferromagnetic ground state. This behavior is in contrast to easy-axis skyrmion hosts such as $\mathrm{GaV}_{4} \mathrm{~S}_{8}$, where the anisotropy is sufficiently weak to shift the skyrmion phase boundaries but not suppress them entirely [14]. In the $R 3 m$ phase, the DMI overcomes the anisotropy energy and leads to the formation of equilibrium spin cycloids at the low energy wavevectors shown in Figure 6(b). The cycloids can form in any direction in the $(a b)$-plane, which is the plane perpendicular to the polar $(c)$ axis of the $R 3 m$ structure.

The low-temperature magnetic behavior of the $R 3 \mathrm{~m}$ and $I m m 2$ phases can be summarized using a conventional micromagnetic free energy density functional with micromagnetic parameters given in Table I, which is directly comparable to that of other skyrmion-host materials [8, 37]:

$$
E=A \sum_{i j} m_{i, j}^{2}+\sum_{i j k n} D_{k n} \varepsilon_{i j k} m_{i} m_{j, n}+K_{u} m_{u}^{2}
$$

Here, $m$ is a magnetization unit vector, $A$ is the exchange stiffness, $D_{k n}$ is the Dzyaloshinkii coupling, and $K_{u}$ is the uniaxial anisotropy constant defined with respect to the $c$-axis of the $R 3 m$ phase, and the $b$-axis of the $I m m 2$ phase. The indices $i j k n$ iterate over Cartesian directions $(x, y, z)$, and $\varepsilon_{i j k}$ is the Levi-Civita symbol. The notation $m_{i, j}$ denotes a partial derivative $\partial m_{i} / \partial r_{j}$. The orientation of the $R 3 m$ and $I m m 2$ crystals with respect to the Cartesian axes is given in Supplementary Figure S4 [26]. A detailed description of this functional can be found in the literature $[36,38]$.

Having established the low-temperature, zero-field magnetic behavior of the two phases comprising our $\mathrm{GaMo}_{4} \mathrm{Se}_{8}$ sample, we proceed to construct the full field $v s$. temperature phase diagram of the material. We focus on the $R 3 m$ phase, as shown in Figure 6(c), because the Imm2 phase remains a uniaxial ferromagnet at all fields within our experimental range and does not contribute to the observed phase transitions. For fields oriented along the $(a b)$-plane, the phase diagram is defined by a transition from the cycloidal ground state to a field-polarized ferromagnetic state near $\mu_{0} H=0.1 \mathrm{~T}$. Below $\approx T_{C} / 3$, this phase transition is first order, while at higher temperatures it proceeds as a second order transition through a canted cycloid intermediate. When the field is oriented along the $c$-axis, the low-field cycloid undergoes a first-order transition into a skyrmion lattice phase at around $\mu_{0} H=0.3 \mathrm{~T}$, which appears to remain stable to very high fields, up to $\mu_{0} H=1.7 \mathrm{~T}$. Magnetic order persists up to $T_{C}=32 \mathrm{~K}$, with a fluctuation-disordered Brazovskii region appearing immediately above $T_{C}$. While some quantitative discrepancies exist between this computational phase diagram and the experimental data, in particular in the range of fields for which skyrmions appear to be stable, the overall trends in phase behavior are informative for determining which phase transitions are being observed in our powder data.

The trends in phase behavior observed in the computational model confirm that the the high-entropy region observed in the magnetoentropic map likely corresponds to a skyrmion phase in $R 3 \mathrm{~m}$ grains with the $c$-axis nearly parallel to the applied field. Furthermore, we propose that the low field transition seen in the susceptibility data is from a cycloidal to a ferromagnetic state in $R 3 \mathrm{~m}$ grains where the magnetic field is mostly aligned perpendicular to the polar axis. The high field transition is most likely a transition from the skyrmion lattice to a ferromagnetic state in $R 3 \mathrm{~m}$ grains in which the magnetic field is mostly aligned along the polar $(c)$ axis. Finally, the $\operatorname{Imm} 2$ phase contributes a ferromagnetic background, with no additional magnetic phase transitions. The similar magnitude of the exchange energy between the $I m m 2$ and $R 3 \mathrm{~m}$ phases, approximated as $A$ in Table I, suggests that their magnetic ordering temperatures should be quite similar, which explains why two magnetic ordering transitions are not seen in the experimental data.

To verify the presence of long-wavelength magnetic order in $\mathrm{GaMo}_{4} \mathrm{Se}_{8}$, we performed powder SANS measurements at $15 \mathrm{~K}$ in the regions we predict to correspond to cycloid and skyrmion stability. The points at which SANS data was taken are indicated on our pro- 
(a)

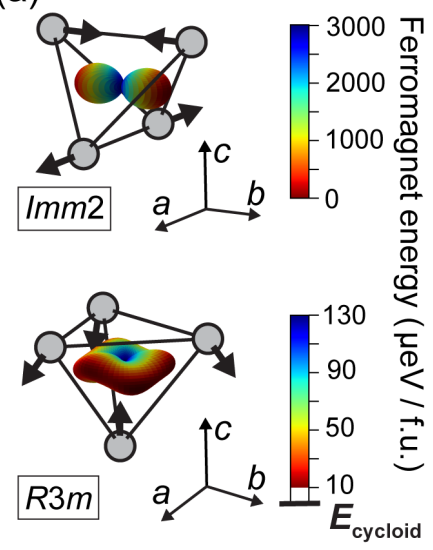

(b)

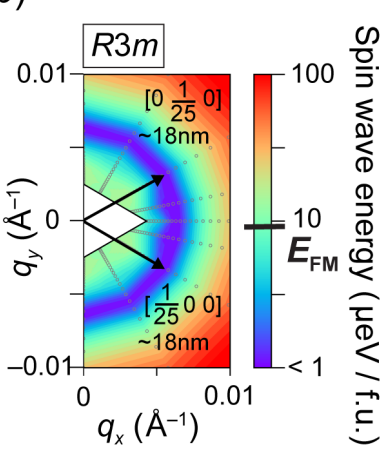

(c)

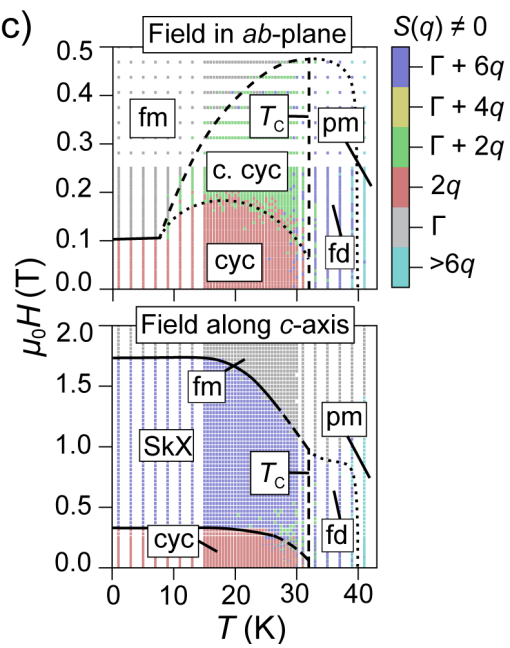

FIG. 6. (a) Computed magnetocrystalline anisotropy in the $I m m 2$ and $R 3 m$ phases of $\mathrm{GaMo}_{4} \mathrm{Se}_{8}$. Grey circles denote Mo atoms, and black arrows illustrate the direction of the Jahn-Teller distortion required to form each phase. The plotted color and distance from the tetrahedron center denote the energy of a ferromagnetic spin configuration oriented along the corresponding crystallographic direction. (b) Energy of spin wave configurations in the (ab)-plane of the $R 3 m$ phase as a function of their $q$-vector, revealing the family of cycloid ground states with a predicted wavelength of $\approx 18 \mathrm{~nm}$. (c) Computed magnetic phase diagram of the $R 3 \mathrm{~m}$ phase for fields oriented along the $c$-axis or the $(a b)$-plane. Color denotes the magnetic structure factor $S(q)$, while phase boundaries are drawn to best capture discontinuities in structure factor, suscetibility, heat capacity, and magnetization. Phase labels correspond to cycloid (cyc), canted cycloid (c. cyc), skyrmion (SkX), ferromagnet (fm), fluctuation disordered (fd), and paramagnet $(\mathrm{pm})$ regions. Solid and dashed lines denote first and second order phase transitions respectively, while dotted lines denote a transition that is continuous within the resolution of our data.

posed phase diagram in Figure 5(a) with triangle markers. Due to the small moment of $1 \mu_{B} / \mathrm{Mo}_{4}$ cluster and different grains in different magnetic phases, the signal is quite low. Nonetheless, after subtracting background data taken at $T=30 \mathrm{~K}$ (above the Curie temperature), we see one to two peaks in the SANS signal at all applied fields, shown in Figure 5(c), corresponding to a real-space magnetic periodicity of around $16 \mathrm{~nm}$, in acceptable agreement with our theoretical calculations for cycloid/skyrmion periodicity $(18 \mathrm{~nm})$.

\section{DISCUSSION}

We have established that $\mathrm{GaMo}_{4} \mathrm{Se}_{8}$ undergoes a complex structural phase transition due to a relatively flat energy landscape in which multiple low temperature space groups have similar energies, as well as a competition between phases favored thermodynamically and kinetically. While the $\mathrm{GaV}_{4}(\mathrm{~S} / \mathrm{Se})_{8}$ compounds exhibit only the $R 3 m$ structure at low temperature, $\mathrm{GaMo}_{4} \mathrm{Se}_{8}$ undergoes a structural transition from the high temperature $F \overline{4} 3 \mathrm{~m}$ phase into two coexisting polar space groups, rhombohedral $R 3 \mathrm{~m}$, which is the ground state, and orthorhombic $\operatorname{Im} m 2$, which is metastable. In the lacunar spinel family, $\mathrm{GeV}_{4} \mathrm{~S}_{8}$ undergoes a Jahn-Teller distortion into an Imm2 structure, likely because it has one extra electron in the $t_{2}$ orbital relative to $\mathrm{GaV}_{4} \mathrm{~S}_{8}$, stabilizing a different splitting of the $t_{2}$ orbital [39]. We speculate that in $\mathrm{GaMo}_{4} \mathrm{Se}_{8}$, the increased filling of the $t_{2}$ orbital relative to the V- compounds, along with enhanced spin-orbit coupling, creates an energy landscape with the $\operatorname{Imm} 2$ and $R 3 \mathrm{~m}$ structures much closer in energy than in other members of the family.

The competition between the stable and metastable crystal structures of $\mathrm{GaMo}_{4} \mathrm{Se}_{8}$ could be used to tune the multiferroic properties of $\mathrm{GaMo}_{4} \mathrm{Se}_{8}$, for instance by straining the material in such a way to stabilize one of the crystal structures. Our computational studies shows that the slight differences between the $\operatorname{Im} m 2$ and $R 3 m$ structures have a dramatic impact on the magnetic properties due to a large change in magnetocrystalline anisotropy. Thus, any strain which alters the relative stability of the two phases will in turn have a strong impact on magnetic behavior. Specifically, stabilization of the $I m m 2$ phase leads to uniaxial ferromagnetism, while favoring the $R 3 \mathrm{~m}$ phase leads to long-wavelength magnetic order with a cycloidal ground state and Néel-type skyrmions. The strong emergent magnetostructural coupling in $\mathrm{GaMo}_{4} \mathrm{Se}_{8}$ provides a route towards the mechanical control of exotic magnetism.

It is worth noting that extra diffraction peaks similar to those of the $I m m 2$ phase in $\mathrm{GaMo}_{4} \mathrm{Se}_{8}$ were also observed in the low temperature diffraction patterns of the sulfide equivalent, $\mathrm{GaMo}_{4} \mathrm{~S}_{8}$ and attributed to coexistence of the cubic phase [11]. Thus, it is possible that $\mathrm{GaMo}_{4} \mathrm{~S}_{8}$ could also have coexisting rhombohedral and orthorhombic low temperature structures, and exhibit similarly strong emergent magnetostructural coupling. Given the recent exciting magnetic discoveries 
in $\mathrm{GaMo}_{4} \mathrm{~S}_{8}[16]$, the discovery of an $\operatorname{Imm} 2$ phase in $\mathrm{GaMo}_{4} \mathrm{Se}_{8}$ merits a re-examination of the crystal structure evolution of $\mathrm{GaMo}_{4} \mathrm{~S}_{8}$ with synchrotron resolution.

Finally, we have shown that the $R 3 \mathrm{~m}$ phase of $\mathrm{GaMo}_{4} \mathrm{Se}_{8}$ has a magnetic phase diagram with longwavelength magnetic phases stable over a wide temperature and field range. $\mathrm{GaMo}_{4} \mathrm{Se}_{8}$ has a higher Curie temperature than the V-compounds or $\mathrm{GaMo}_{4} \mathrm{~S}_{8}$ at $T_{C}=27.5 \mathrm{~K}$, with cycloids and Néel-type skyrmions stable down to the lowest temperatures measured $(T=2 \mathrm{~K})$. With a field applied along the polar axis, cycloids are stable from $\mu_{0} H=0 \mathrm{~T}$ to around $\mu_{0} H=0.15 \mathrm{~T}$ and skyrmions are stable up to $\mu_{0} H=0.25 \mathrm{~T}$ at $T=5 \mathrm{~K}$. SANS data show a magnetic periodicity of around $16 \mathrm{~nm}$, in good agreement with our computational analysis $(18 \mathrm{~nm})$. Overall, the qualitative form of the phase diagram is in good agreement between our experimental results, computational analysis, and previous reports in the literature for materials of similar symmetry[14, 4042]. However, there are deviations from experiment in the exact range of fields for which skyrmions are stable. The discrepancy in the cycloid to skyrmion transition is of a similar magnitude to the error in the Curie temperature and cycloid to in-plane ferromagnet transition, and is most likely a result of errors in the underlying DFT representation of $\mathrm{GaMo}_{4} \mathrm{Se}_{8}$ magnetism, as well as deviations in the $g$-factor from our assumed value of 2 . The much larger discrepancy in the upper bound on skyrmion stability versus applied field is more likely a result of stray fields, which we neglect in our simulations. Finally, while we do not observe the complexity in magnetic ordering vector reported in $\mathrm{GaMo}_{4} \mathrm{~S}_{8}$ [16], this difference may be a consequence of the sensitivity of data we are able to obtain from a powder sample as compared to a single crystal.

\section{CONCLUSION}

A combined experimental and computational investigation of the low-temperature structural and magnetic phase behavior of the $\mathrm{GaMo}_{4} \mathrm{Se}_{8}$ lacunar spinel has been carried out. We show that this material undergoes a Jahn-Teller distortion at $T=51 \mathrm{~K}$ into two co-existing structures - a metastable Imm 2 configuration and the ground state $R 3 \mathrm{~m}$ phase. These two phases both order magnetically at $T_{C}=27.5 \mathrm{~K}$, but exhibit dramatically different magnetic behavior due to differences in their magnetocrystalline anisotropy. While the $\operatorname{Imm} 2$ phase is found from computation to be a uniaxial ferromagnet, the $R 3 \mathrm{~m}$ phase favors a $16 \mathrm{~nm}$ cycloid ground state, and a Néel-skyrmion lattice under applied field. We conclude that the coexistence of these two phases with dramatically different magnetic properties makes $\mathrm{GaMo}_{4} \mathrm{Se}_{8}$ a promising material for realizing mechanical control over exotic magnetism, as small energy differences separating the $I m m 2$ and $R 3 m$ phases translate into a very large change in magnetic behavior.

\section{ACKNOWLEDGMENTS}

This research was supported by the National Science Foundation (NSF) under DMREF Award DMR-1729489. Partial support from the NSF Materials Research Science and Engineering Center (MRSEC) at UC Santa Barbara, DMR-1720256 (IRG-1) is gratefully acknowledged (D. A. K. and A.V.dV.) Use of the Shared Experimental Facilities of the UCSB MRSEC (NSF DMR 1720256) is acknowledged. The UCSB MRSEC is a member of the NSF-supported Materials Research Facilities Network (www.mrfn.org). We also acknowledge support from the Center for Scientific Computing (NSF DMR-1720256 and NSF CNS-1725797), as well as the National Energy Research Scientific Computing Center, a DOE Office of Science User Facility supported by DOE DE-AC0205CH11231. Use of the Advanced Photon Source at Argonne National Laboratory was supported by the U. S. Department of Energy, Office of Science, Office of Basic Energy Sciences, under Contract No. DE-AC0206CH11357. We thank Dr. S. Lapidus of 11-BM at the Advanced Photon Source for helpful contributions. We acknowledge the support of Dr. M. Bleuel and the National Institute of Standards and Technology, U.S. Department of Commerce, in providing the neutron research facilities used in this work.
[1] I. I. Mazin, H. O. Jeschke, K. Foyevtsova, R. Valenti, and D. I. Khomskii, $\mathrm{Na}_{2} \mathrm{IrO}_{3}$ as a Molecular Orbital Crystal, Phys. Rev. Lett. 109, 197201 (2012).

[2] Z. Hiroi, Structural Instability of the Rutile Compounds and its Relevance to the Metal-Insulator Transition of $\mathrm{VO}_{2}$, Progr. Solid State Chem. 43, 47 (2015).

[3] S. V. Streltsov and D. I. Khomskii, Covalent Bonds Against Magnetism in Transition Metal Compounds, Proc. Natl. Acad. Sci. 113, 10491 (2016).

[4] E. C. Schueller, J. L. Zuo, J. D. Bocarsly, D. A. Kitchaev, S. D. Wilson, and R. Seshadri, Modeling the Structural Distortion and Magnetic Ground State of the Polar Lacunar Spinel $\mathrm{GaV}_{4} \mathrm{Se}_{8}$, Phys. Rev. B 100, 045131 (2019).
[5] H.-S. Kim, J. Im, M. J. Han, and H. Jin, Spin-Orbital Entangled Molecular $j_{\text {eff }}$ States in Lacunar Spinel Compounds, Nat. Commun. 5, 3988 (2014).

[6] Y. Fujima, N. Abe, Y. Tokunaga, and T. Arima, Thermodynamically Stable Skyrmion Lattice at Low Temperatures in a Bulk Crystal of Lacunar Spinel $\mathrm{GaV}_{4} \mathrm{Se}_{8}$, Phys. Rev. B 95, 180410(R) (2017).

[7] E. Ruff, A. Butykai, K. Geirhos, S. Widmann, V. Tsurkan, E. Stefanet, I. Kezsmarki, A. Loidl, and P. Lunkenheimer, Polar and Magnetic Order in $\mathrm{GaV}_{4} \mathrm{Se}_{8}$, Phys. Rev. B 96, 165119 (2017).

[8] S. Bordacs, A. Butykai, B. G. Szigeti, J. S. White, R. Cubitt, A. O. Leonov, S. Widmann, D. Ehlers, H.-A. K. von 
Nidda, V. Tsurkan, A. Loidl, and I. Kezsmarki, Equilibrium Skyrmion Lattice Ground State in a Polar Easy-plane Magnet, Sci. Rep. 7, 7584 (2017).

[9] R. Pocha, D. Johrendt, and R. Pottgen, Electronic and Structural Instabilities in $\mathrm{GaV}_{4} \mathrm{~S}_{8}$ and $\mathrm{GaMo}_{4} \mathrm{~S}_{8}$, Chem. Mater. 12, 2882 (2000).

[10] M. Francois, O. V. Alexandrov, K. Yvon, H. B. YaichAerrache, P. Gougeon, M. Potel, and M. Sergent, Structural Phase Transition in $\mathrm{GaMo}_{4} \mathrm{Se}_{8}$ and $\mathrm{AlMo}_{4} \mathrm{~S}_{8}$ by Xray Powder Diffraction, Z. Kristallogr. Cryst. Mater. 200, 47 (1992).

[11] M. Francois, W. Lengauer, and K. Yvon, Structural Phase Transition in $\mathrm{GaMo}_{4} \mathrm{~S}_{8}$ by X-ray Powder Diffraction, Z. Kristallogr. Cryst. Mater. 196, 111 (1990).

[12] A. K. Rastogi, A. Berton, J. Chaussy, R. Tournier, M. Potel, R. Chevrel, and M. Sergent, Itinerant Electron Magnetism in the $\mathrm{Mo}_{4}$ Tetrahedral Cluster Compounds $\mathrm{GaMo}_{4} \mathrm{~S}_{8}$, $\mathrm{GaMo}_{4} \mathrm{Se}_{8}$, and $\mathrm{GaMo}_{4} \mathrm{Se}_{4} \mathrm{Te}_{4}$, J. Low Temp. Phys. 52, 539 (1983).

[13] A. K. Rastogi and E. P. Wohlfarth, Magnetic FieldInduced Transitions in the $\mathrm{Mo}_{4}$ Cluster Compounds $\mathrm{GaMo}_{4} \mathrm{~S}_{8}$ and $\mathrm{GaMo}_{4} \mathrm{Se}_{8}$ Showing Heavy Fermion Behaviour, Phys. Stat. Sol. (b) 142, 569 (1987).

[14] D. A. Kitchaev, E. C. Schueller, and A. Van der Ven, Mapping Skyrmion Stability in Uniaxial Lacunar Spinel Magnets from First-Principles, Phys. Rev. B 101, 054409 (2020).

[15] M. Y. Jeong, S. H. Chang, B. H. Kim, J.-H. Sim, A. Said, D. Casa, T. Gog, E. Janod, L. Cario, S. Yunoki, M. J. Han, and J. Kim, Direct Experimental Observation of the Molecular $J_{\text {eff }}=3 / 2$ Ground State in the Lacunar Spinel $\mathrm{GaTa}_{4} \mathrm{Se}_{8}$, Nat. Commun. 8, 782 (2017).

[16] A. Butykai, D. Szaller, L. F. Kiss, L. Balogh, M. Garst, L. DeBeer-Schmitt, T. Waki, Y. Tabata, H. Nakamura, I. Kezsmarki, and S. Bordacs, Squeezing Magnetic Modulations by Enhanced Spin-Orbit Coupling of $4 d$ Electrons in the Polar Semiconductor $\mathrm{GaMo}_{4} \mathrm{~S}_{8}$, arXiv 1910.11523 (2019).

[17] G. Kresse and J. Furthmuller, Efficient Iterative Schemes for Ab Initio Total-Energy Calculations Using a PlaneWave Basis Set, Phys. Rev. B 54, 11169 (1996).

[18] P. E. Blochl, Projector Augmented-Wave Method, Phys. Rev. B 50, 17953 (1994).

[19] G. Kresse and D. Joubert, From Ultrasoft Pseudopotentials to the Projector Augmented-Wave Method, Phys. Rev. B 59, 1758 (1999).

[20] J. P. Perdew, K. Burke, and M. Ernzerhof, Generalized Gradient Approximation Made Simple, Phys. Rev. Lett. 77, 3865 (1996).

[21] B. J. Campbell, H. T. Stokes, D. E. Tanner, and D. M. Hatch, ISODISPLACE: a web-based tool for exploring structural distortions, J. Appl. Cryst. 39, 607 (2006).

[22] S. P. Ong, W. D. Richards, A. Jain, G. Hautier, M. Kocher, S. Cholia, D. Gunter, V. L. Chevrier, K. A. Persson, and G. Ceder, Python Materials Genomics (pymatgen): A Robust, Open-Source Python Library for Materials Analysis, Comput. Mater. Sci. 68, 314 (2013).

[23] R. Drautz and M. Fähnle, Spin-Cluster Expansion: Parametrization of the General Adiabatic Magnetic Energy Surface with ab initio Accuracy, Phys. Rev. B 69, 104404 (2004).

[24] J. C. Thomas and A. Van der Ven, The Exploration of Nonlinear Elasticity and its Efficient Parameterization for Crystalline Materials, J. Mech. Phys. Solids 107, 76
(2017)

[25] A. Van der Ven, J. C. Thomas, B. Puchala, and A. R. Natarajan, First-Principles Statistical Mechanics of Multicomponent Crystals, Annu. Rev. Mater. Res. 48, 27 (2018).

[26] See Supplemental Material at [URL will be inserted by publisher] for $\mathrm{AC}$ and $\mathrm{dM} / \mathrm{dT}$ magnetic data, as well as details of the magnetic cluster expansion and magnetic Hamiltonians used in this work..

[27] A. Van de Walle and G. Ceder, Automating First-Principles Phase Diagram Calculations, J. Phase Equilib. 23, 348 (2002).

[28] S. Jakob, Strukturen, Magnetismus und Phasenumwandlungen der Mott-Isolatoren $G a\left(M_{4-x} M_{x}^{\prime}\right) Q_{8}$ und $\left(M_{4-x} M_{x}^{\prime}\right) Q_{4} I_{4}\left(M, M^{\prime}=M o, N b, \mathrm{Ta} ; Q=S\right.$, Se; $x=0-4)$, Ph.D. thesis, Ludwig-Maximilians-Universitat Munchen (2010).

[29] A. A. Coelho, TOPAS and TOPAS-Academic: An Optimization Program Integrating Computer Algebra and Crystallographic Objects Written in $\mathrm{C}++$, J. Appl. Crystallogr. 51, 210 (2018).

[30] K. Momma and F. Izumi, VESTA3 for Three-Dimensional Visualization of Crystal, Volumetric and Morphology Data, J. Appl. Crystallogr. 44, 1272 (2011).

[31] S. R. Kline, Reduction and Analysis of SANS and USANS Data Using IGOR Pro, J. Appl. Cryst. 39, 895 (2006).

[32] J. D. Bocarsly, R. F. Need, R. Seshadri, and S. D. Wilson, Magnetoentropic Signatures of Skyrmionic Phase Behavior in FeGe, Phys. Rev. B 97, 100404(R) (2018).

[33] M. C. Kemei, P. T. Barton, S. L. Moffitt, M. W. Gaultois, J. A. Kurzman, R. Seshadri, M. R. Suchomel, and Y.-I. Kim, Crystal Structures of Spin-Jahn-Teller-Ordered $\mathrm{MgCr}_{2} \mathrm{O}_{4}$ and $\mathrm{ZnCr}_{2} \mathrm{O}_{4}$, J. Phys.: Condens. Matter 25, 326001 (2013).

[34] J. D. Bocarsly, C. Heikes, C. M. Brown, S. D. Wilson, and R. Seshadri, Deciphering Structural and Magnetic Disorder in the Chiral Skyrmion Host Materials $\mathrm{Co}_{x} \mathrm{Zn}_{y} \mathrm{Mn}_{z}$ $(x+y+z=20)$, Phys. Rev. Materials 3, 014402 (2019).

[35] L. Kautzsch, J. D. Bocarsly, C. Felser, S. D. Wilson, and R. Seshadri, Controlling Dzyaloshinskii-Moriya Interactions in the Skyrmion Host Candidates $\mathrm{FePd}_{1-x} \mathrm{Pt}_{x} \mathrm{Mo}_{3} \mathrm{~N}$, Phys. Rev. Materials 4, 024412 (2020).

[36] D. A. Kitchaev, I. J. Beyerlein, and A. Van der Ven, Phenomenology of Chiral Dzyaloshinskii-Moriya Interactions in Strained Materials, Phys. Rev. B 98, 214414 (2018).

[37] R. Takagi, D. Morikawa, K. Karube, N. Kanazawa, K. Shibata, G. Tatara, Y. Tokunaga, T. Arima, Y. Taguchi, Y. Tokura, and S. Seki, Spin-Wave Spectroscopy of the Dzyaloshinskii-Moriya Interaction in Room-Temperature Chiral Magnets Hosting Skyrmions, Phys. Rev. B 95, 220406(R) (2017).

[38] C. Abert, Micromagnetics and Spintronics: Models and Numerical Methods, Eur. Phys. J. B 92, 120 (2019).

[39] D. Bichler, V. Zinth, D. Johrendt, O. Heyer, M. K. Forthaus, T. Lorenz, and M. M. Abd-Elmeguid, Structural and Magnetic Phase Transitions of the $\mathrm{V}_{4}$-cluster compound $\mathrm{GeV}_{4} \mathrm{~S}_{8}$, Phys. Rev. B 77, 212102 (2008).

[40] A. Bogdanov and A. Hubert, Thermodynamically Stable Magnetic Vortex States in Magnetic Crystals, J. Magn. Magn. Mater. 138, 255 (1994).

[41] U. Güngördü, R. Nepal, O. A. Tretiakov, K. Belashchenko, and A. A. Kovalev, Stability of Skyrmion Lattices and Symmetries of Quasi-Two-Dimensional Chiral Magnets, Phys. Rev. B 93, 064428 (2016). 
[42] J. Rowland, S. Banerjee, and M. Randeria, Skyrmions in Chiral Magnets with Rashba and Dresselhaus Spin-Orbit Coupling, Phys. Rev. B 93, 020404(R) (2016). 


\title{
Structural evolution and skyrmionic phase diagram of the lacunar spinel $\mathrm{GaMo}_{4} \mathrm{Se}_{8}$
}

\author{
Emily C. Schueller, ${ }^{1}$ Daniil A. Kitchaev, ${ }^{1}$ Julia L. Zuo, ${ }^{1}$ Joshua D. Bocarsly, ${ }^{1}$ \\ Joya A. Cooley, ${ }^{1}$ Anton Van der Ven, ${ }^{1}$ Stephen D. Wilson, ${ }^{1}$ and Ram Seshadri ${ }^{1,2}$ \\ ${ }^{1}$ Department of Materials and Materials Research Laboratory, \\ University of California, Santa Barbara, Santa Barbara, CA, 93106 \\ ${ }^{2}$ Department of Chemistry and Biochemistry, \\ University of California, Santa Barbara, Santa Barbara, CA, 93106
}




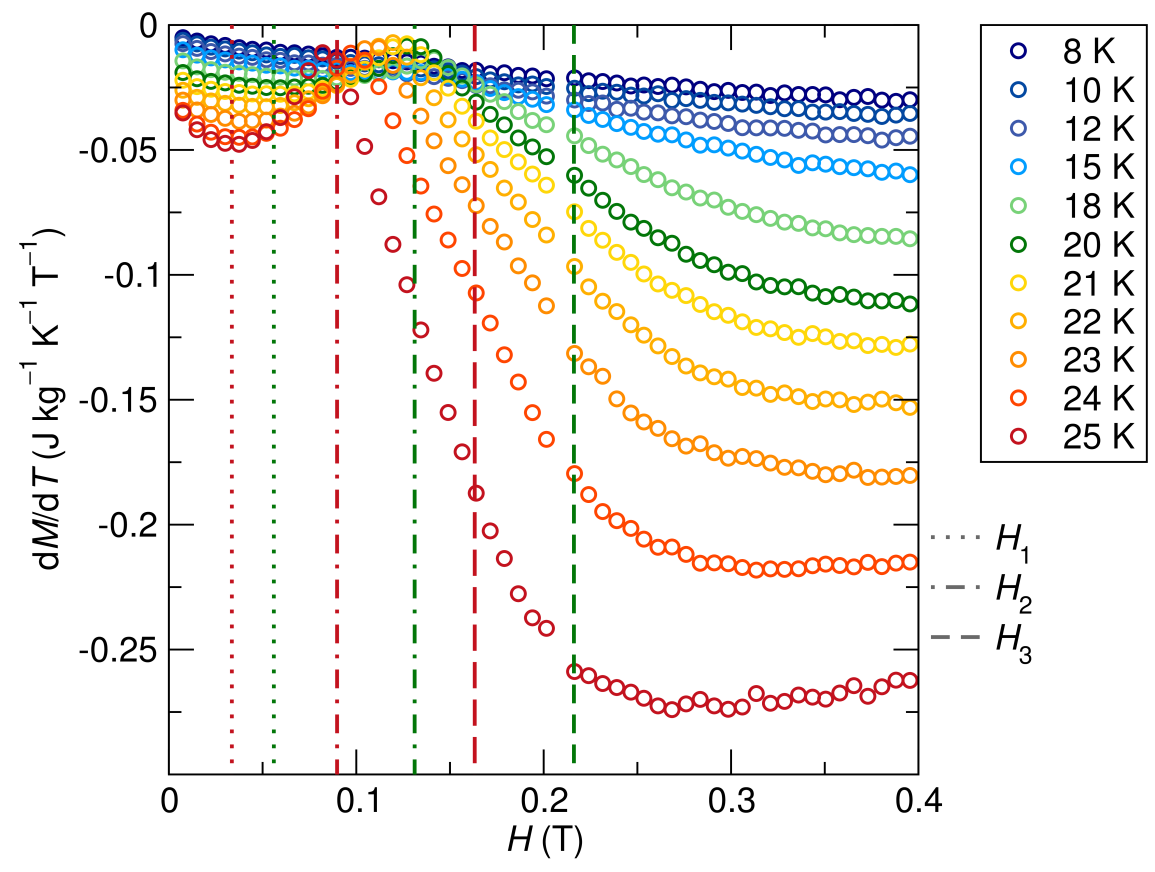

FIG. S1. $\mathrm{d} M / \mathrm{d} T$ vs field data at various temperatures. Some characteristic locations of the three magnetic transitions with field are shown over the data.

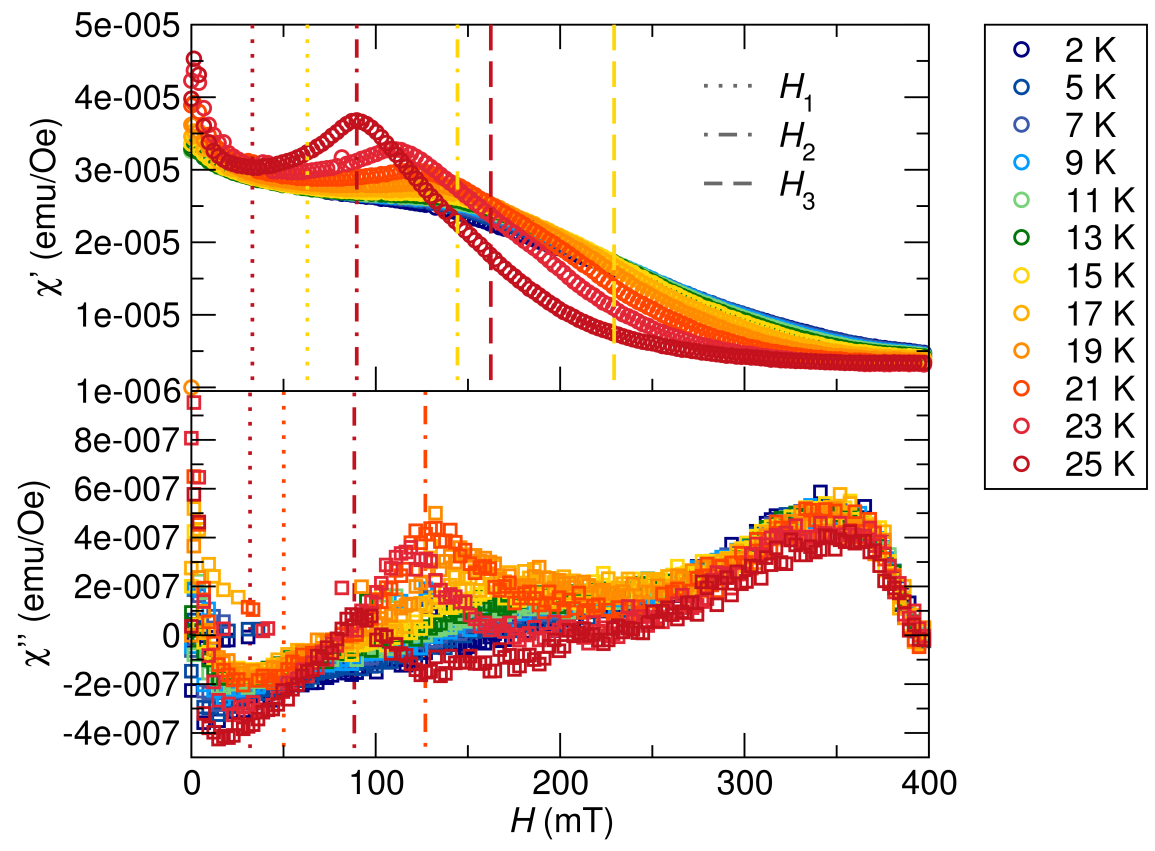

FIG. S2. AC $\chi^{\prime}$ and $\chi^{\prime \prime}$ data at various temperatures. Some characteristic locations of the three magnetic transitions with field are shown over the data. Only the first two transitions can be elucidated from the $\chi "$ data. 


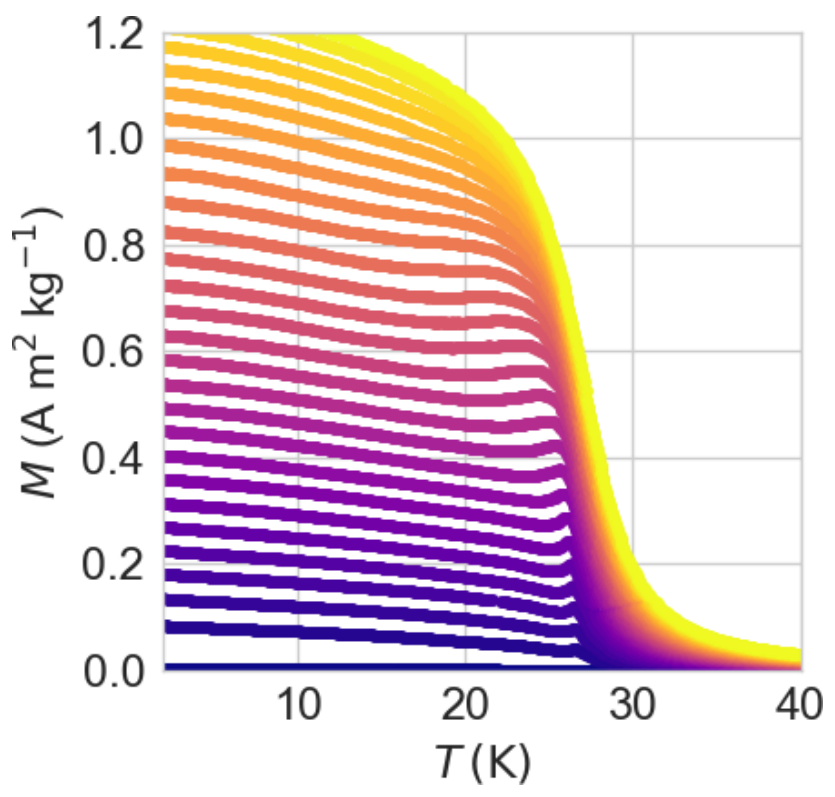

FIG. S3. Magnetization vs temperature data at various applied fields. This data was used to generate the magnetoentropic map by taking the derivative of magnetization with respect to temperature.

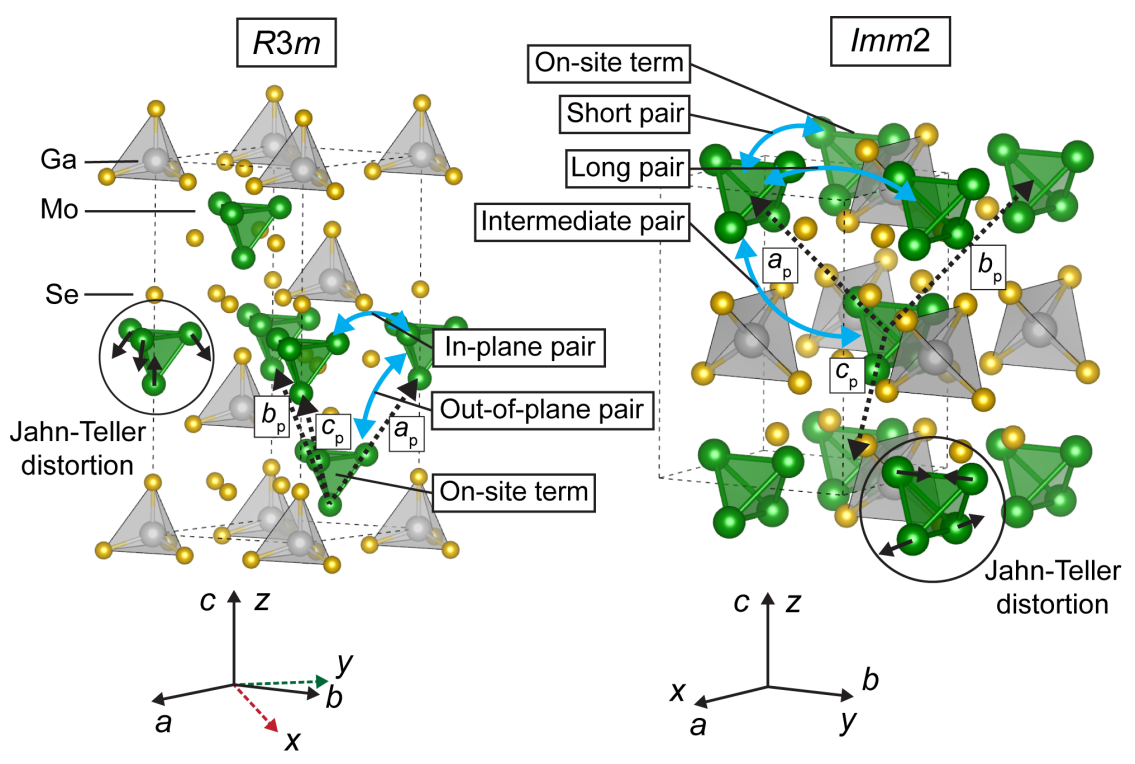

FIG. S4. Structure of the $R 3 m$ and $I m m 2$ phases of $\mathrm{GaMo}_{4} \mathrm{Se}_{8}$ in their conventional unit cells, along with the primitive cell axes $\left(a_{\mathrm{p}}, b_{\mathrm{p}}, c_{\mathrm{p}}\right)$. The cartesian coordinate system for each phase further defines the axes used to define the magnetic Hamiltonian. Finally, the symmetrically distinct on-site and pair interactions appearing in the magnetic Hamiltonian of each phase are shown. 

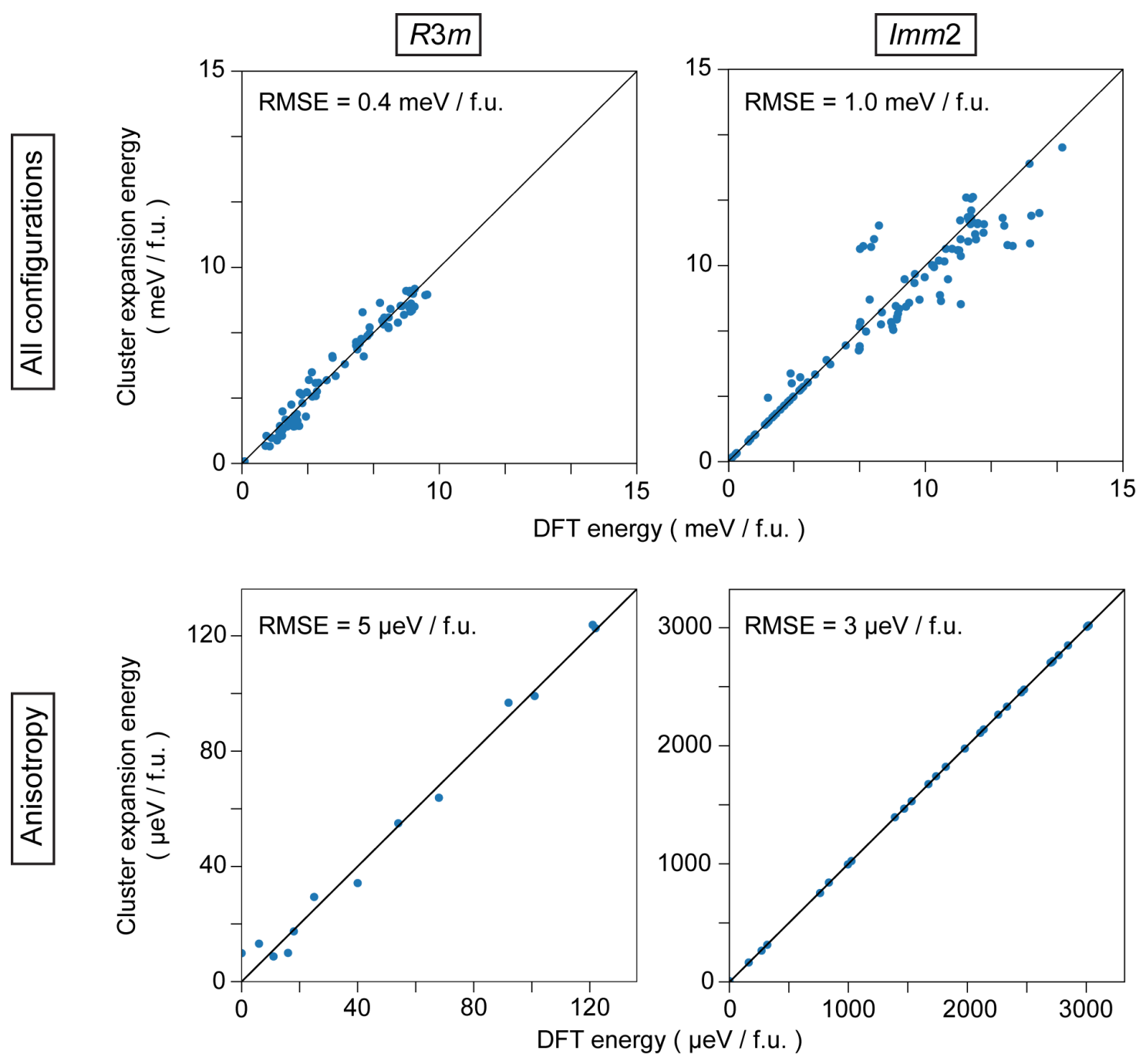

FIG. S5. Magnetic cluster expansion fitting error for the $R 3 m$ and $I m m 2$ phases of $\mathrm{GaMo}_{4} \mathrm{Se}_{8}$. Each point denotes the energy of a symmetrically distinct spin configuration, as computed using DFT and the fitted cluster expansion Hamiltonian. The magnetocrystalline anisotropy terms are fitted independently to account for the difference in energy scale between these terms and spin-spin interactions, as described in the main text. 


\begin{tabular}{|c|c|c|c|}
\hline Cluster type & & Basis function & $J(\mathrm{meV})$ \\
\hline On-site & $\phi_{1}^{A}$ & $\sqrt{2}|2,0\rangle$ & $0.027(1)$ \\
\hline$r=(0,0,0)$ & $\phi_{2}^{A}$ & $-i(|4,-3\rangle+|4,3\rangle)$ & $-0.008(1)$ \\
\hline (1 equiv.) & $\phi_{3}^{A}$ & $\sqrt{2}|4,0\rangle$ & $-0.006(1)$ \\
\hline Out-of-plane & $\phi_{7}^{E}$ & $\frac{\sqrt{2}}{3}|1,1 ; 0,0\rangle$ & $1.38(6)$ \\
\hline$r_{1}=(0,0,0)$ & $\phi_{8}^{D}$ & $\frac{i}{3}(|1,1 ; 1,1\rangle-|1,1 ; 1,-1\rangle)$ & $-0.06(9)$ \\
\hline$r_{2}=(-1,0,0)$ & $\phi_{9}^{A}$ & $-\frac{i}{3}(|1,1 ; 2,1\rangle+|1,1 ; 2,-1\rangle)$ & $0.1(2)$ \\
\hline \multirow[t]{2}{*}{ (3 equiv.) } & $\phi_{10}^{A}$ & $\frac{\sqrt{2}}{3}|1,1 ; 2,0\rangle$ & 0 \\
\hline & $\phi_{11}^{A}$ & $\frac{1}{3}(|1,1 ; 2,2\rangle+|1,1 ; 2,-2\rangle)$ & $0.6(3)$ \\
\hline In-plane & $\phi_{12}^{E}$ & $\frac{\sqrt{2}}{3}|1,1 ; 0,0\rangle$ & $1.06(6)$ \\
\hline$r_{1}=(0,0,0)$ & $\phi_{13}^{D}$ & $\left(\frac{1}{6}+\frac{i}{\sqrt{12}}\right)|1,1 ; 1,1\rangle+$ & $0.3(1)$ \\
\hline$r_{2}=(-1,0,1)$ & & $\left(\frac{1}{6}-\frac{i}{\sqrt{12}}\right)|1,1 ; 1,-1\rangle$ & \\
\hline \multirow[t]{6}{*}{ (3 equiv.) } & $\phi_{14}^{D}$ & $\frac{-i \sqrt{2}}{3}|1,1 ; 1,0\rangle$ & 0 \\
\hline & $\phi_{15}^{A}$ & $\frac{\sqrt{2}}{3}|1,1 ; 2,0\rangle$ & 0 \\
\hline & $\phi_{16}^{A}$ & $\left(\frac{1}{\sqrt{12}}-\frac{i}{6}\right)|1,1 ; 2,1\rangle-$ & 0 \\
\hline & & $\left(\frac{1}{\sqrt{12}}+\frac{i}{6}\right)|1,1 ; 2,-1\rangle$ & \\
\hline & $\phi_{17}^{A}$ & $\left(\frac{1}{6}-\frac{i}{\sqrt{12}}\right)|1,1 ; 2,2\rangle+$ & 0 \\
\hline & & $\left(\frac{1}{6}+\frac{i}{\sqrt{12}}\right)|1,1 ; 2,-2\rangle$ & \\
\hline
\end{tabular}

TABLE I. Magnetic Hamiltonian for the $R 3 m$ phase of $\mathrm{GaMo}_{4} \mathrm{Se}_{8}$, consisting of basis functions $\phi$ and parametrized interaction strengths $J$. Cluster site coordinates and basis functions are given for the reference cluster, in lattice coordinates with respect to the primitive lattice vectors $\left(a_{\mathrm{p}}, b_{\mathrm{p}}, c_{\mathrm{p}}\right)$ given in Supplementary Figure 3. The number of equivalents for each cluster refers to the number of symmetrically-equivalent clusters of this type per primitive cell. Basis functions are defined in terms of spherical harmonics $|l, m\rangle=\sqrt{4 \pi} Y_{m}^{l}(\phi, \theta)$ for the on-site terms and Clebsch-Gordan functions $\left|l_{1}, l_{2} ; L, M\right\rangle=4 \pi \sum_{m_{1}, m_{2}} c_{m_{1}, m_{2}, M}^{l_{1}, l_{2}, L} Y_{m_{1}}^{l_{1}}\left(\phi_{1}, \theta_{1}\right) Y_{m_{2}}^{l_{2}}\left(\phi_{2}, \theta_{2}\right)$ for pair clusters $\left(r_{1}, r_{2}\right)$. Spin angles $(\phi, \theta)$ are given in spherical coordinates with respect to the global coordinate system defined in Supplementary Figure 4. Basis function superscripts denote whether the interaction corresponds to exchange (E), DMI (D), or anisotropy (A). Parenthesis in the $J$ vector components denote uncertainty in the last digit. 


\begin{tabular}{|c|c|c|c|}
\hline Cluster type & & Basis function & $J(\mathrm{meV})$ \\
\hline On-site & $\phi_{1}^{A}$ & $\sqrt{2}|2,0\rangle$ & $0.313(1)$ \\
\hline$r=(0,0,0)$ & $\phi_{2}^{A}$ & $|2,2\rangle+|2,-2\rangle$ & $0.551(1)$ \\
\hline \multirow[t]{3}{*}{ (1 equiv.) } & $\phi_{3}^{A}$ & $|4,4\rangle+|4,-4\rangle$ & $-0.003(1)$ \\
\hline & $\phi_{4}^{A}$ & $|4,2\rangle+|4,-2\rangle$ & $-0.002(1)$ \\
\hline & $\phi_{5}^{A}$ & $\sqrt{2}|4,0\rangle$ & 0 \\
\hline Short & $\phi_{10}^{E}$ & $\sqrt{2}|1,1 ; 0,0\rangle$ & $0.93(7)$ \\
\hline$r_{1}=(0,0,0)$ & $\phi_{11}^{D}$ & $|1,1 ; 1,1\rangle+|1,1 ; 1,-1\rangle$ & $-0.18(9)$ \\
\hline$r_{2}=(0,-1,-1)$ & $\phi_{12}^{A}$ & $|1,1 ; 2,-2\rangle+|1,1 ; 2,-2\rangle$ & $-0.2(2)$ \\
\hline (1 equiv.) & $\phi_{13}^{A}$ & $\sqrt{2}|1,1 ; 2,0\rangle$ & $-0.2(1)$ \\
\hline Intermediate & $\phi_{14}^{E}$ & $\frac{\sqrt{2}}{4}|1,1 ; 0,0\rangle$ & $0.2(1)$ \\
\hline$r_{1}=(1,0,0)$ & $\phi_{15}^{D}$ & $\frac{1}{4}(|1,1 ; 1,1\rangle+|1,1 ; 1,-1\rangle)$ & $0.3(2)$ \\
\hline$r_{2}=(0,-1,-1)$ & $\phi_{16}^{D}$ & $\frac{i}{4}(|1,1 ; 1,1\rangle-|1,1 ; 1,-1\rangle)$ & $-0.1(1)$ \\
\hline \multirow[t]{6}{*}{ (4 equiv.) } & $\phi_{17}^{D}$ & $\frac{-i \sqrt{2}}{4}|1,1 ; 1,0\rangle$ & $-0.3(2)$ \\
\hline & $\phi_{18}^{A}$ & $\frac{1}{4}(|1,1 ; 2,2\rangle+|1,1 ; 2,-2\rangle)$ & $0.2(3)$ \\
\hline & $\phi_{19}^{A}$ & $\frac{i}{4}(|1,1 ; 2,2\rangle-|1,1 ; 2,-2\rangle)$ & $-0.1(4)$ \\
\hline & $\phi_{20}^{A}$ & $\frac{-1}{4}(|1,1 ; 2,1\rangle-|1,1 ; 2,-1\rangle)$ & $0.4(5)$ \\
\hline & $\phi_{21}^{A}$ & $\frac{-i}{4}(|1,1 ; 2,1\rangle+|1,1 ; 2,-1\rangle)$ & $0.5(5)$ \\
\hline & $\phi_{22}^{A}$ & $\frac{\sqrt{2}}{4}|1,1 ; 2,0\rangle$ & $0.1(1)$ \\
\hline Long & $\phi_{23}^{E}$ & $\sqrt{2}|1,1 ; 0,0\rangle$ & $0.76(8)$ \\
\hline$r_{1}=(1,0,0)$ & $\phi_{24}^{D}$ & $i(|1,1 ; 1,-1\rangle-|1,1 ; 1,1\rangle)$ & $0.24(9)$ \\
\hline$r_{2}=(0,0,-1)$ & $\phi_{25}^{A}$ & $|1,1 ; 2,2\rangle+|1,1 ; 2,-2\rangle$ & $-0.1(1)$ \\
\hline (1 equiv.) & $\phi_{26}^{A}$ & $\sqrt{2}|1,1 ; 2,0\rangle$ & $0.1(1)$ \\
\hline
\end{tabular}

TABLE II. Magnetic Hamiltonian for the $I m m 2$ phase of $\mathrm{GaMo}_{4} \mathrm{Se}_{8}$, following the format given in the caption of Supplementary Table 1. 